\title{
Neuroimmunology of Disordered Sleep in Depression and Alcoholism
}

\author{
Michael Irwin, M.D.
}

The specific functions of sleep are not known, although sleep is commonly considered a restorative process that is important for the proper functioning of the immune system. Severity of disordered sleep in depressed and alcoholic subjects correlates with declines in natural and cellular immunity and is associated with alterations in the complex cytokine network. Despite evidence that sleep and sleep loss have effects on immune processes and nocturnal secretion of cytokines, the physiological significance of these immune changes is not known. Moreover, in view of basic evidence of a reciprocal interaction between sleep and cytokines, further research is needed to understand whether alterations in cytokines contribute to disordered sleep.

[Neuropsychopharmacology 25:S45-S49, 2001] (C) 2001 American College of Neuropsychopharmacology. Published by Elsevier Science Inc.
KEY WORDS: Sleep; Immunity; Cytokines; Major depression; Alcoholism

Sleep is implicated in the homeostatic regulation of the neuroendocrine, autonomic nervous and immune systems, and is hypothesized to have a restorative function on immune processes (Horne 1988). Normal sleep is believed to have a role in the proper functioning of natural and cellular immune responses, whereas sleep loss leads to impairment of host defense mechanisms possibly impacting susceptibility to viral and bacterial pathogens (Benca and Quintas 1997; Dinges et al. 1995). Despite the importance of understanding the relationships between sleep and immunity with implications for disease risk, the role of sleep in normal immune functioning has not been extensively examined in humans. Moreover, there are virtually no data that have evaluated the association between disordered sleep and

From the Department of Psychiatry and Biobehavioral Sciences, University of California, Los Angeles, CA.

Address correspondence to: Michael Irwin, M.D. Norman Cousins Professor, Cousins Center for Psychoneuroimmunology, 300 UCLA Medical Plaza, Suite 3-109, UCLA School of Medicine, Los Angeles, CA 90095, Tel.: (310) 825-8281; Fax: (310) 794-7247; email mirwin1@ucla.edu immunity in psychiatric patient populations, even though such subjects show prominent disturbances of sleep (Benca et al. 1992) and are at risk for infectious and other immune-related diseases (Cook 1998; Irwin 1999).

In addition to the hypothesized role of sleep in the modulation of immune function, further evidence indicates a bi-directional communication between sleep and the immune system. Animal studies have found that the complex cytokine network influences sleep and sleep depth (Krueger and Toth 1994). Indeed, cytokines, which are secreted by immune cells to regulate immune processes, have both somnogenic and inhibitory effects depending on the cytokine, dose, and circadian phase (Krueger and Toth 1994; Benca and Quintas 1997). However, translation of these basic mechanisms into the clinic has not been carried forward despite the tremendous implications of such studies for answering why sleep may be disordered in certain patient populations such as depressed and alcoholic subjects.

Thus, the objective of this review is to examine the relationships between disturbances of sleep and immunity in humans by assessing the association between disordered sleep and immune alterations that coincide in depression and alcoholism. 


\section{DEPRESSION, SLEEP AND IMMUNITY}

Depression serves as an excellent model to learn more about sleep-immune interactions as depressed patients exhibit prominent abnormalities of sleep (Benca et al. 1992), dysregulation of the neuroendocrine and sympathetic nervous systems and alterations of immunity such as a reduction of lymphocyte responses and natural killer (NK) activity (Irwin 1999). Peripheral blood NK cell activity is an in vitro measure of cellular immune function that correlates with host resistance to viral illness (Trinchieri 1989). However, not all studies of stressed and/or depressed patients have found alterations of NK activity and it is likely that changes of cytotoxicity are related to depressed mood or depressive symptom severity (Irwin 1999).

\section{Relationship between Subjective Sleep Disturbance and Natural Killer Cell Activity}

The relationship between clinical depression, symptom severity and NK activity might be explained in part by the association of depression with specific behaviors that modulate immune responses. Depressed patients show disturbances in sleep, changes in activity levels, and alterations in appetite and possibly weight. Thus, to evaluate the association between severity of depressive symptoms and NK activity, immune measures were taken in acutely depressed patients who were free of anti-depressant medication at the time of assessment. Severity of depression was measured using total and subscale Hamilton Depression Rating Scale scores (Cover and Irwin 1994). Values of NK activity were negatively correlated with the subscales retardation $(\mathrm{r}=-0.34, p<.05)$ and sleep disturbance $(\mathrm{r}=-0.33, p<$ .05). In addition, correlations using individual sleep disturbance items demonstrated that early insomnia, but not middle or late insomnia, was negatively correlated with cytotoxicity $(\mathrm{r}=-0.40, p<.01)$. Neither Hamilton total scores nor any of the other subscales (anxiety/somatization, weight loss, cognitive disturbance or diurnal variation) was related to NK activity in depressed patients. In other words, clinical symptoms of sleep disturbance and retardation are distinctly associated with values of NK activity, and depressed patients who report insomnia and/or retardation are more likely to show depression-related reduction of NK cytotoxicity.

Independent of the severity of depressive symptoms and the presence of a depressive disorder, it is also possible that sleep has a distinct role in the modulation of immune function. Consistent with this hypothesis, insomnia and NK activity were also found to be negatively correlated in alcoholics even when the variance accounted for by other Hamilton depressive symptoms and drinks per drinking day was statistically controlled (Irwin 1999). In addition, we have found that individu- als who are undergoing a severe life event or major difficulty show a reduction of natural cytotoxicity even though they do not have clinically significant depressive symptoms (Irwin 1999). Importantly, these nondepressed stressed subjects with reduced levels of NK activity report insomnia, and thus it appears that insomnia is associated with reduced natural cytotoxicity even in subjects in whom other depressive symptoms are minimal.

\section{Objective EEG Sleep and Immunity}

To extend observations that demonstrate an association between subjective complaints of insomnia and reduced NK activity, the relationships between objective EEG sleep parameters and NK cytotoxicity were evaluated (Irwin et al. 1992). Insomnia is characterized by disturbance of sleep EEG continuity measures including increased sleep latency and decreased total sleep time and sleep efficiency (Benca et al. 1992). Furthermore, this decrement in amount and continuity of sleep is accounted for by a loss of nonREM sleep. Thus, if subjective insomnia is associated with immunity, then sleep continuity EEG parameters and amount of nonREM are hypothesized to correlate with immune function. For example, if depressed patients show increased sleep latency and reduced total sleep time, sleep efficiency, and nonREM sleep, then we predict that sleep latency will negatively correlate with NK activity, whereas total sleep time, sleep efficiency, and duration of nonREM sleep will positively correlate with NK cytotoxicity. In addition, since insomnia is associated with NK activity independent of severity of depressive symptoms (Cover and Irwin 1994), similar correlations between sleep continuity measures and NK activity are hypothesized in non-depressed control subjects.

Consistent with these predictions, sleep amounts and continuity measures were associated with NK activity. In depressed patients, total sleep time, sleep efficiency, and duration of nonREM sleep were positively correlated with lytic activity. In addition, Stage 2 sleep was positively correlated with NK activity (Irwin et al. 1992). In controls, similar relationships were found which indicate that associations between disordered sleep and immunity were independent of the effects of a mood disorder. Again, total sleep time, sleep efficiency, and duration of nonREM sleep were positively correlated with NK activity in the controls similar to the relationships in the depressives. These data extend our previous findings of an association between insomnia and natural cytotoxicity in depression and further demonstrate that EEG sleep amounts and continuity measures are associated with immune function independent of the presence of a mood disorder.

Sleep is regulated by a multitude of substances interwoven with each other which are also capable of affect- 
ing immune function (Krueger and Toth 1994), and we speculate that both sleep processes and natural cytotoxicity might be modulated by a common central factor. For example, the neuropeptide corticotropin releasing factor is increased in the cerebrospinal fluid of depressed patients, inhibits normal sleep and interleukin-1 induced sleep, and acts within the central nervous system to activate sympathetic outflow and reduce cellular immunity. Conversely, interleukin-1 induces slow wave sleep in animals (Krueger and Toth 1994), is increased in the plasma of humans following the onset of slow wave sleep (Moldofsky et al. 1986), and acts on NK cells to stimulate cytolytic activity. Future studies are needed to evaluate whether the secretory pattern of cytokines, or some other peptide with sleep-immune effects, is altered in depressed patients to produce coincident changes of sleep and immunity.

\section{ALCOHOLISM, SLEEP AND IMMUNITY}

Sleep disturbance is one of the most common complaints of alcoholic patients. (Benca et al. 1992) Alcohol dependent patients report difficulty falling asleep and staying asleep and EEG sleep is fragmented, shallow, and short in duration during alcohol withdrawal, even in the absence of delirium tremens (DTs), convulsions, or other major withdrawal syndrome. Furthermore, during the first month or so of abstinence, alcoholics show a reduction of total sleep time, fragmentation of sleep, and loss of delta sleep as compared with agematched controls.

Age and severity of alcohol dependence correlate with abnormal sleep in alcoholics, and recent data indicate that ethnicity is a critical factor in mediating the effects of alcohol dependence on sleep (Irwin et al. 2000). African-American ethnicity and alcohol dependence interact to produce a more profound loss of delta sleep as measured by polysomnographic and spectral sleep analyses than that found in Euro-American alcoholics (Figure 1). The amounts of Stage 3, Stage 4 and delta sleep were reduced in the alcoholic subjects versus controls. Furthermore, the African-American subjects showed the greatest loss of delta sleep as compared with the other three groups (Figure 1). Indeed, none of the African-American subjects had measurable amounts of Stage 4 sleep when scored by raters blind to the group status of the EEG records.

Spectral analyses of the sleep EEG confirmed the loss of delta sleep in the alcohol dependent patients. As compared with the controls, alcoholics showed significantly lower delta $(0.75-4.5 \mathrm{~Hz})$ activity over the whole night and especially during the first NREM period. Significant reductions in mean spectral power over the entire frequency spectrum $(0.75-40 \mathrm{~Hz})$ were also found

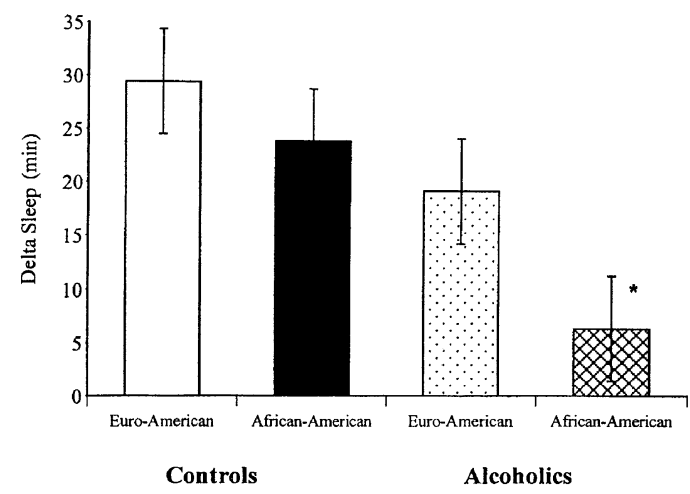

Figure 1. Delta sleep (minutes). Data are presented as mean \pm SEM. There are 17 Euro-American controls and 14 African-American controls, 17 Euro-American alcoholics, and 14 African-American alcoholics. *Planned comparisons found that African-American alcoholics showed the greatest loss of delta sleep as compared with the other three groups $(\mathrm{F}=4.3, p<.03)$.

in alcoholics for the whole night and during the first NREM period.

Given the hypothesis that sleep contributes to the maintenance of the immune system, (Dinges et al. 1995; Horne 1988), additional studies were conducted to evaluate whether African-American alcoholics who are at increased risk for disordered sleep are more likely to show immunological disturbances as compared with the other groups. The effects of chronic alcohol dependence on NK and cellular immune responses were examined in the four groups of alcoholic and control subjects described above. Liver disease, current alcohol or other drug consumption, and/or concurrent withdrawal symptoms or medications were not present which might otherwise confound the alcoholic subjects' immunological status. Assessment of immune function was extended beyond assay of natural immunity and included an evaluation of levels of pro-inflammatory cytokine (IL-6) and the balance of Th-1 like (IL-2, IL-12) and Th2 like (IL-10) cytokines. In humans, two polar categories of T helper (Th) cells are described: Th1 cells secrete IL-2, IL-12 and interferon- $\gamma$ (IFN) which predominantly stimulate cell-mediated responses, whereas Th2 cells secrete IL-4 and IL-10 which mainly stimulate antibody-mediated responses. Changes in the relative expression of Th1 to Th2 cytokines may have important clinical implications in alcoholics. For example, alcoholics show a high incidence of hepatitis $C$, and the chronicity of this viral infection is related to a lack of Th1 cytokine production and/or an increase in Th2 release. For example, Woitas et al. (1997) have found that hepatitis C seropositive blood donors without viremia show increased Th1 cytokine production (IFN, IL-2) in response to hepatitis $\mathrm{C}$ core protein, whereas seropositive donors with viremia show increases in IL-10-producing T cells. 
In parallel with the increased risk of disordered sleep in African-American alcoholics, these subjects also had significantly lower levels of NK activity than the other three groups (Figure 2). Similarly, IL-2 stimulated NK activity was lower in African-American alcoholics as compared with levels in controls and white alcoholics. Evaluation of stimulated cytokine production also revealed differences in which the African-American alcoholics showed the greatest changes. Production of the pro-inflammatory cytokine, IL-6, was significantly lower in the African-American alcoholics as compared with the other three groups (Figure 3), whereas production of the inhibitory cytokine IL-10 was significantly increased in the African-American alcoholics as compared with controls and white alcoholics (Figure 4). There were no significant differences between groups for IL-2 and IL-12.

In summary, alcohol dependence and African-American ethnicity jointly contribute to changes of delta sleep which coincide with alterations of NK cell responses and stimulated IL-6 and IL-10 production. First, African-American alcoholics show the greatest decline of slow wave sleep activity as assessed by EEG and spectral analyses. Second, African-American alcoholic subjects showed the greatest decline of NK activity and stimulated NK activity along with alterations in the pattern of stimulated cytokine production. Peripheral blood mononuclear cell production of the proinflammatory cytokine IL- 6 was reduced, whereas the negative immunoregulatory Th2 cytokine IL-10 was increased in the African-American alcoholics.

The model put forward in this review posits an interrelationship between alcohol dependence, sleep, and cytokine expression in which feedback systems are operating. Not only is disordered sleep thought to impact

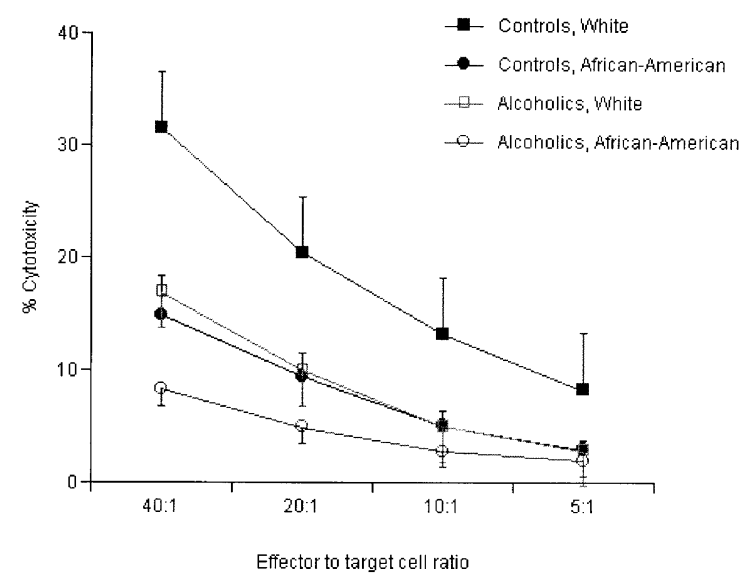

Figure 2. Natural killer cell activity across the four effector-to-target cell ratios in white controls (ם), African-American controls (O), white alcoholics $(\square)$, and AfricanAmerican alcoholics $(\bigcirc)$. Data are presented as mean \pm SEM. There are 17 white controls, 14 African-American controls, 17 white alcoholics, and 14 African-American alcoholics.

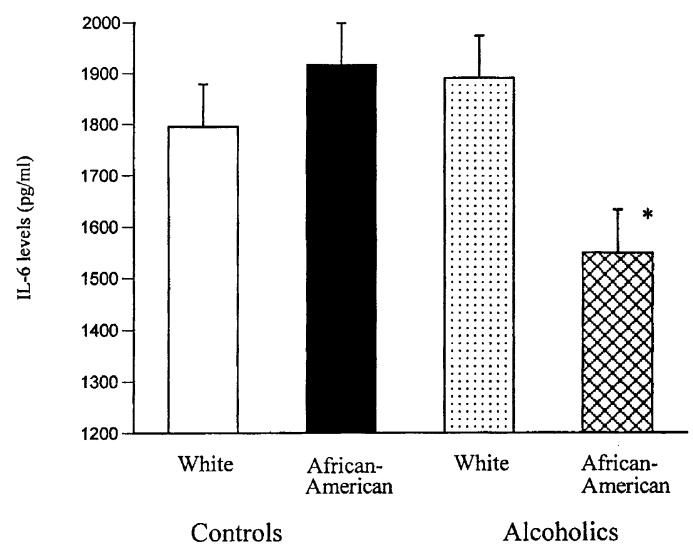

Figure 3. Con-A stimulated production of IL-6 by peripheral blood mononuclear cells in control and alcoholic patients stratified as white and African-American subjects. Data are presented as mean \pm SEM. There are 16 white controls, 14 African-American controls, 16 white alcoholics, and 14 African-American alcoholics. *Supernatant levels of IL-6 were significantly lower in the African-American alcoholics as compared with the other three groups $(\mathrm{F}=7.2, p<.01)$.

sleep, but alternatively cytokines are hypothesized to alter sleep depth and intensity. In animals, IL-6, TNF, IFN and IL-10 are involved in the physiological regulation of sleep (Krueger and Toth 1994). For example, pro-inflammatory cytokines such as IL-1 and TNF increase delta sleep (Kapás et al. 1992; Opp et al. 1991), whereas IL-10 inhibits slow wave sleep in animals (Opp et al. 1995). IL-6 also decreases nonREM sleep, although this action was small due to the use of a cross-species

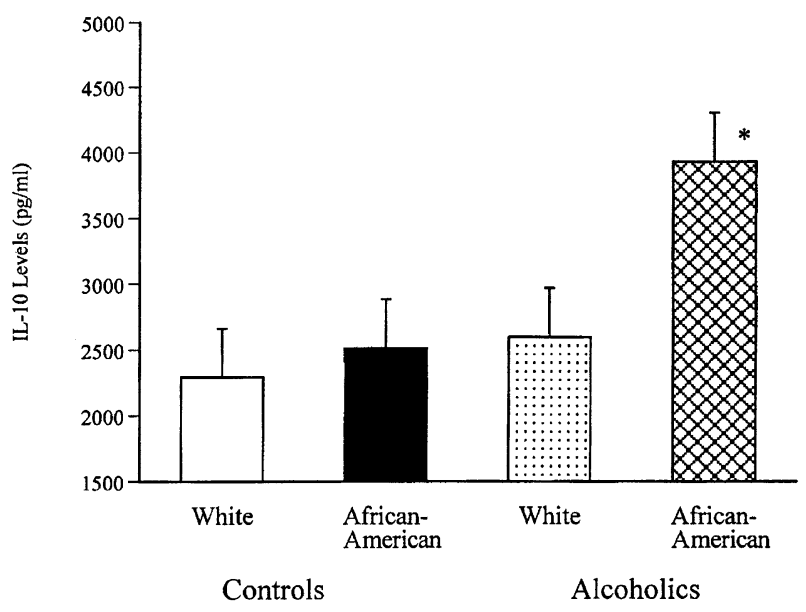

Figure 4. Con-A stimulated production of IL-10 by peripheral blood mononuclear cells in control and alcoholic patients stratified as white and African-American subjects. Data are presented as mean \pm SEM. There are 16 white controls, 14 African-American controls, 16 white alcoholics, and 14 African-American alcoholics. *Supernatant levels of IL-10 were significantly increased in the African-American alcoholics as compared with controls and white alcoholics. ( $\mathrm{F}=$ $4.9, p<.05)$. 
form of IL-6 (Opp et al. 1989). However, in humans, acute peripheral administration of IL-6 reduces delta sleep in the first half of the night when circulating levels of IL-6 are elevated (Späth-Schwalbe et al. 1998). Vgontzas and colleagues have further found that daytime elevations of circulating IL-6 are associated with severity of sleepiness and fatigue in sleep disordered patients (Vgontzas et al. 1997), and that sleep deprivation leads to daytime over-secretion of IL-6 (Vgontzas et al. 1999) in which daytime plasma levels of IL-6 negatively correlate with amounts of slow wave sleep. In view of this converging evidence, it is tempting to speculate about a link between cytokines and disordered sleep in the African-American alcoholics in which cytokine abnormalities contribute the severity of sleep disturbance in these patients.

\section{CONCLUSIONS}

Sleep loss is endemic in society, and loss of sleep during only part of the night is one of the most common complaints of persons who experience environmental or psychological stress, travel across time meridians, engage in shift work, or suffer from a psychiatric disorder. In clinical samples of depressed or alcoholic subjects, disordered sleep correlates with impairments in immune functioning. These data indicate that sleep disturbance has to be taken into account as a cause of immune alterations or as a factor exacerbating them. However, the ultimate question about whether sleep compromises subjects' health remains unanswered. None of the studies have employed disease specific immune measures nor intensively monitored changes in health status in relationship to administration of a sleep deprivation paradigm. Experimental approaches that involve administration of a viral challenge or biologically relevant immunization that is coupled with sleep deprivation are needed to test the impact of sleep on clinically relevant endpoints.

\section{ACKNOWLEDGMENTS}

This work was supported in part by NIAAA grant AA10215, NIMH grant 5T32-18399, NIMH grant 2P30MH30914, and NIH grant M01 RR00827.

\section{REFERENCES}

Benca RM, Obermeyer WH, Thisted RA, Gillin JC (1992): Sleep and psychiatric disorders: a meta analysis. Arch Gen Psych 49:651-668

Benca RM, Quintas J (1997): Sleep and host defenses: a review. Sleep 20:1027-1037

Cook RT (1998): Alcohol abuse, alcoholism, and damage to the immune system-A review. Alc Clin Exp Res 22:1927-1942
Cover H, Irwin M (1994): Immunity and depression: insomnia,retardation, and reduction of natural killer cell activity. J Beh Med 17:217-223

Dinges DF, Douglas SD, Hamarman S, Zaugg L, Kapon S (1995): Sleep deprivation and human immune function. Adv Neuroimmunol 5:97-110

Horne J (1988): Why We Sleep: The Function of Sleep in Humans and Other Mammals. Oxford, Oxford University Press.

Irwin M (1999): Immune correlates of depression. Adv Exp Med Biol 461:1-24

Irwin M, Miller C, Gillin JC, Demodena A, Ehlers C (2000): Polysomnographic and spectral sleep EEG in primary alcoholics: an interaction between alcohol dependence and African-American ethnicity. Alc Clin Exp Res 24:1376-1384

Irwin M, Smith TL, Gillin JC (1992): Electroencephalographic sleep and natural killer activity in depressed patients and control subjects. Psychosom Med 54:10-21

Kapás L, Hong L, Cady AB, Opp MR, Postlethwaite AE, Seyer JM, Krueger JM (1992): Somnogenic, pyrogenic, and anorectic activities of tumor necrosis factor-alpha and TNF-alpha fragments. Am J Physiol 263:R708-R715

Krueger JM, Toth LA (1994): Cytokines as regulators of sleep. Ann NY Acad Sci 739:299-310

Moldofsky H, Lue FA, Eisen J, Keystone E, Gorczynski RM (1986): The relationship of interleukin-1 and immune functions to sleep in humans. Psychosom Med 48:309318

Opp M, Obal F Jr, Cady AB, Johannsen L, Krueger JM (1989): Interleukin-6 is pyrogenic but not somnogenic. Physiol Beh 45:1069-1072

Opp MR, Obal F Jr, Krueger JM (1991): Interleukin 1 alters rat sleep: temporal and dose-related effects. Am J Physiol 260:R52-R58

Opp MR, Smith EM, Hughes TK (1995): Interleukin-10 (cytokine synthesis inhibitory factor) acts in the central nervous system of rats to reduce sleep. J Neuroimmunol 60:165-168

Späth-Schwalbe E, Hansen K, Schmidt F, Schrezenmeier H, Marshall L, Burger K, Fehm HL, Born J (1998): Acute effects of recombinant human interleukin-6 on endocrine and central nervous sleep functions in healthy men. J Clin Endocrin Metab 83:1573-1579

Trinchieri G (1989): Biology of natural killer cells. Adv Immunol 47:187-376

Vgontzas AN, Papanicolaou DA, Bixler EO, Kales A, Tyson K, Chrousos GP (1997): Elevation of plasma cytokines in disorders of excessive daytime sleepiness: role of sleep disturbance and obesity. J Clin Endocrin Metab 82: 1313-1316

Vgontzas AN, Papanicolaou DA, Bixler EO, Lotsikas A, Zachman K, Kales A, Prolo P, Wong ML, Licinio J, Gold PW, Hermida RC, Mastorakos G, Chrousos GP (1999): Circadian interleukin-6 secretion and quantity and depth of sleep. J Clin Endocrin Metab 84:26032607

Woitas RP, Lechmann M, Jung G, Kaiser R, Sauerbruch T, Spengler U (1997): CD30 induction and cytokine profiles in hepatitis $C$ virus core-specific pheripheral blood T lymphocytes. J Immunol 159:1012-1018 